\title{
Impacto de la simulación clínica en el nivel de confianza para evaluar pacientes en estudiantes de Medicina
}

\author{
Impact of clinical simulation on the level of confidence \\ to evaluate patients in medical students \\ Erik Manuel Camacho-Zúñiga,* Melany Galván-Estrada, ${ }^{\ddagger}$ \\ Daniel Chávez-De La Rosa, $\$$ Yushlin Arylei Estrada,\| \\ Verenice Zarahí González-Mejía
}

\begin{abstract}
Palabras clave:
Simulación, nivel confianza, alumnos, medicina.

Keywords: Simulation, confidence level, students, medicine.
\end{abstract}

* Médico pasante del Servicio Social en el Centro de Simulación adscrito a la Universidad de Montemorelos.

₹ Médico pasante del Servicio Social en el Centro de Salud Puebla adscrito a la Secretaría de Salud de Puebla.

$\S$ Profesor adscrito a la Escuela de Medicina FACSA-UM.

" Médico pasante del Servicio Social en Departamento de Apoyo a la Investigación en Ciencias de Salud.

" Coordinación de Investigación, Departamento de Apoyo a la Investigación en Ciencias de Salud.

\section{RESUMEN}

Introducción: La simulación en la educación médica ha tenido un increíble desarrollo a nivel mundial; ésta lleva al alumno a un escenario que lo hace sentir en la realidad. Objetivos: Comparar el nivel de confianza de estudiantes de medicina, para enfrentar al paciente de cardiología en un escenario de urgencias; antes y después de la simulación clínica. Material y métodos: Estudio observacional, comparativo, longitudinal. Participaron estudiantes de la clase de cardiología de una escuela de medicina de una universidad privada. El instrumento utilizado fue Self-Confidence Scale versión en español. Se aplicó el test previo y posterior a la simulación. Se utilizó la prueba estadística de Wilcoxon para variables dependientes ordinales, y para el tamaño del efecto la prueba de Rosenthal. Se usó un muestreo no probabilístico por conveniencia, que incluyó a todos los que cumplían los criterios. Resultados: Fueron 77 alumnos, $42.9 \%$ hombres y $57.1 \%$ mujeres. Se encontró un nivel de confianza mayor en las cuatro dimensiones, después de la simulación $(\mathrm{p}<0.001)$. En los extranjeros, hubo un mayor aumento del nivel posterior a la prueba de simulación ( $\mathrm{p}<$ 0.000). Conclusiones: La simulación clínica aumentó la confianza en los estudiantes de cardiología.

\section{ABSTRACT}

Introduction: Simulation in medical education has had an incredible development worldwide, this takes the student to a scenario that makes him feel in reality. Objectives: To compare the level of confidence of medical students, to face the cardiology patient in an emergency setting; before and after clinical simulation. Material and methods: Observational, comparative, longitudinal study. Students from the cardiology class of a private university medical school participated. The instrument used was SelfConfidence Scale Spanish version. The test was applied before and after the simulation. The Wilcoxon statistical test was used for ordinal dependent variables and the Rosenthal test for effect size. Non-probability sampling was used for convenience including all those who met the criteria. Results: 77 students, $42.9 \%$ male and $57.1 \%$ female were included. A higher level of confidence was found in the 4 dimensions after simulation $(p<0.001)$. In foreigners there was a greater increase in the level of confidence following the simulation test $(p<0.000)$. Conclusions: Clinical simulation increased the level of confidence in medical students in cardiology.

\section{INTRODUCCIÓN}

$E^{n}$ la educación médica, la simulación lleva al alumno a un escenario donde se crean situaciones o casos parecidos a los que deberá enfrentar con pacientes, en una realidad clínica. ${ }^{1,2}$

El Instituto Nacional de Medicina de Estados Unidos de América informó que hasta 98,000 muertes al año sucedían por errores médicos, más que por algunas enfermedades de alta prevalencia. ${ }^{3}$ En un estudio sobre prevalencia de eventos adversos en cinco países de Latinoamérica (México, Perú, Argentina, Costa Rica y Colombia), que involucró a 58 centros y el análisis total de 11,555 pacientes hospitalizados; $60 \%$ de los eventos adversos encontrados se consideraron evitables: $28.69 \%$ con algún procedimiento y $6.15 \%$ con el diagnóstico. ${ }^{4}$

Según un artículo publicado en la red de Revistas Científicas de América Latina, el Caribe, España y Portugal, existen cerca de 400,000 muertes anuales por errores médicos, lo cual es 
un número similar al de muertes por tabaquismo, alcohol, drogas, heridos por arma de fuego y accidentes automovilísticos. Si se suman todos estos factores, la posibilidad de morir en un hospital es 10 veces mayor que en otro lugar. ${ }^{5}$

Es por ello que se deben idear métodos efectivos de educación médica, identificar los puntos débiles y reforzarlos. La educación no puede ser únicamente teórica, debe ser practicada por los estudiantes en su periodo de formación. Es relevante conocer cómo la simulación clínica da al estudiante la oportunidad de pensar en diversos momentos de su aprendizaje: por adelantado, durante la acción y en retrospectiva; con la finalidad de razonar, deliberar y emitir juicios con objetivos y fines establecidos para autorregular su aprendizaje. ${ }^{6,7}$

En México, surgieron algunos centros para la enseñanza de la reanimación cardiopulmonar básica y avanzada; uno de los escenarios clínicos más básicos e importantes que el médico debe dominar. ${ }^{8}$

La simulación promete enriquecer la realización de procedimientos $y$, con ello, disminuir complicaciones y mejorar la supervivencia del paciente. ${ }^{9}$ En el ámbito de cardiología, se requiere adquirir el conocimiento, habilidades y destrezas que permitan manejar las diversas situaciones para reconocer signos, síntomas, gravedad del paciente y poder ser resolutivos apropiadamente. Sin la práctica correspondiente previa, se puede tratar un paciente sin un nivel de confianza apropiado, y ser vulnerable de cometer más errores y negligencias.

En el área de cardiología, se han desarrollado múltiples simuladores como Harvey, para que el estudiante, residente, adscrito y personal de enfermería adquieran conocimientos y destrezas necesarios para la evaluación y tratamiento de situaciones clínicas. ${ }^{10}$ En otro estudio, se observó que los alumnos que realizaron sesiones de simulación en cardiología fueron mejores que el grupo control en el examen teórico y en el práctico. ${ }^{11}$

Para medir el nivel de confianza, existe en la literatura el instrumento Self-Confidence Scale en su versión en español, donde el evaluado contesta según su percepción para poder atender a un paciente; ${ }^{12}$ dicho instrumento será utilizado en esta investigación, para evaluar a los alumnos previo y posterior a las pruebas de simulación.

El objetivo de esta investigación es comparar el nivel de confianza antes y después de la simulación clínica en los estudiantes de cardiología, pertenecientes a una universidad privada de Nuevo León, México.

\section{MATERIAL Y MÉTODOS}

Estudio observacional, comparativo, longitudinal, prospectivo. Se encuestó un total de 77 alumnos de cuarto año de medicina, de enero a mayo 2019. El trabajo fue aprobado por el Comité de Ética e Investigación de la Universidad de Montemorelos, Nuevo León; con número de registro: 2019-005. Se utilizó un muestreo no probabilístico por conveniencia, el cual incluyó a todos los que cumplían los criterios. Se excluyó a aquéllos que hubieran cursado anteriormente la materia de cardiología o realizado las prácticas de simulación.

Antes de que los alumnos hicieran el proceso de simulación clínica, se acudió a la clase teórica de cardiología. Se explicó el propósito de la investigación y se les invitó a participar. Se entregó el consentimiento informado y el cuestionario de autoconfianza. Después, se realizaron cuatro sesiones de simulación clínica, en las que practicaron el manejo médico del síndrome isquémico coronario agudo (SICA) y el debriefing al finalizar cada sesión. Al terminar las cuatro prácticas, contestaron nuevamente el test de autoconfianza. Las sesiones de simulación consistieron en casos de SICA, donde el alumno intervenía individualmente al simulador de paciente SimMom de Laerdal ${ }^{\circledR}$; según las guías de la American Heart Association/ American College of Cardiology. Cada clase duró 20 minutos, y podían realizarla en cuatro ocasiones diferentes. El tiempo transcurrido de las sesiones prácticas para poder realizar el segundo test fue de dos meses, con un intervalo de dos semanas entre cada una.

El nivel de confianza fue medido con el instrumento Self-Confidence Scale en su versión en español. El test consta de 12 ítems, y las respuestas se encuentran en escala tipo Likert de 5 grados, variando desde "nada seguro" hasta "muy seguro". La más alta puntuación indica un mayor nivel de confianza en el alumno. Con una alfa de Cronbach, según el National Council of State Boards of Nursing, en las escalas pre- y post-test de 0.93 y 0.96 , respectivamente. ${ }^{12}$ La escala evalúa cuatro dimensiones: reconocimiento de signos y síntomas (preguntas 1-3); valoración de la condición del paciente (preguntas 4-6); capacidad de intervención (preguntas 7-9) y evaluación del paciente (preguntas 10-12). ${ }^{13}$

Se utilizó la prueba estadística de Wilcoxon para variables dependientes ordinales en el paquete estadístico SPSS V.23. Para valor estadístico 
significativo, se usó el estándar: $\alpha \leq 0.05$. Para el tamaño del efecto, se empleó la prueba estadística para Wilcoxon-U Mann-Whitney, r de Rosenthal, con interpretación de los valores en términos de Cohen.

\section{RESULTADOS}

Participaron 77 alumnos del cuarto año de medicina; con rango de edad entre 18 y 52 años, de los cuales $42.9 \%$ fueron hombres y $57.1 \%$ mujeres. Se observó que la media global del nivel de confianza pre-simulación fue de 37.75, mientras que en la post-simulación aumentó a 49.28. Se encontró que existe una diferencia estadísticamente significativa en las puntuaciones de las medias de las 12 variables de la escala del nivel de confianza antes y después de las pruebas de simulación, además de un tamaño del efecto grande en términos de Cohen (Tabla 1). Los datos que correspondieron a un mayor tamaño del efecto $(r$ $>0.7$, pertenecen a las variables que evalúan el nivel de confianza, con respecto al dolor torácico y su manejo en los pacientes.

En la variable sociodemográfica "sexo", se encontró que el femenino tuvo mejores resultados en la prueba previa a la simulación en las 12 variables de la escala. Después de haber realizado las pruebas de simulación, en las mujeres hubo una mayor diferencia de las medias, comparado con los hombres; a pesar de que ambos sexos comenzaron con una media casi igual antes de la simulación clínica: hombres 3.12 y mujeres 3.16, se tomó como referencia un valor máximo de 5 puntos (Figura 1).

Respecto a la nacionalidad, tanto en los mexicanos como en los extranjeros, hubo un aumento del nivel de confianza, con una diferencia de medias pre- y post-simulación de 0.85 y 1.18 , respectivamente $(p<0.000)$ (Figura 2).

\section{DISCUSIÓN}

En los estudiantes de la materia de cardiología, las sesiones aumentaron su nivel de confianza, ya que tuvieron la oportunidad de enfrentarse a escenarios similares a los que vivirán durante los siguientes años.

En este trabajo en particular, los casos abarcaron infarto agudo al miocardio con y sin elevación del ST. Mientras se desarrollaron los escenarios, los alumnos pusieron a prueba su habilidad para reconocer a un paciente con infarto agudo al miocardio, al igual que su capacidad para poder evaluar, explorar e intervenirlo, a través de un adecuado tratamiento.

En los resultados globales por puntuación, se obtuvo una diferencia de medias de 11.63 puntos ( $p<0.000$ ), lo cual es acorde a un estudio realizado en la Escuela de Enfermería de la Universidad del Bío-Bío, donde se compararon los niveles de confianza global previo y posterior a la intervención, y se obtuvo una diferencia de medias de 9.7 puntos $(p<0.001) .{ }^{13}$ Otra inves-

Tabla 1: Variables de la escala de autoconfianza, medias pre y postsimulación.

\begin{tabular}{|c|c|c|c|c|c|}
\hline Variables del nivel de confianza & $\begin{array}{c}\text { Media } \\
\text { pre-simulación }\end{array}$ & $\begin{array}{c}\text { Media } \\
\text { post-simulación }\end{array}$ & $\mathrm{Z}^{*}$ & $\mathrm{p}^{* *}$ & $\mathrm{r}^{* * *}$ \\
\hline Reconocer signos y síntomas cardiacos & 3.48 & 4.22 & -5.907 & 0.000 & 0.673 \\
\hline Reconocer signos y síntomas respiratorios & 3.39 & 4.03 & -4.697 & 0.000 & 0.535 \\
\hline Reconocer signos y síntomas neurológicos & 3.38 & 4.13 & -5.478 & 0.000 & 0.624 \\
\hline Evaluar dolor de pecho & 3.17 & 4.40 & -6.827 & 0.000 & 0.778 \\
\hline Evaluar problemas de respiración & 3.17 & 4.08 & -6.036 & 0.000 & 0.688 \\
\hline Evaluar cambios del estado mental & 3.05 & 4.13 & -5.889 & 0.000 & 0.671 \\
\hline Intervenir dolor de pecho & 3.09 & 4.35 & -6.948 & 0.000 & 0.792 \\
\hline Intervenir problemas de respiración & 3.03 & 3.97 & -5.974 & 0.000 & 0.681 \\
\hline Intervenir cambios del estado mental & 2.88 & 3.83 & -5.868 & 0.000 & 0.669 \\
\hline Efectividad de intervención en dolor de pecho & 3.04 & 4.25 & -6.587 & 0.000 & 0.751 \\
\hline Efectividad de intervención de problemas respiratorios & 3.06 & 3.94 & -5.397 & 0.000 & 0.615 \\
\hline Efectividad de intervención en cambio de estado mental & 3.01 & 3.96 & -5.590 & 0.000 & 0.637 \\
\hline
\end{tabular}

* Z = valor de Wilcoxon; ${ }^{* *} \mathrm{p}$ = valor del error alpha, ${ }^{* * *} \mathrm{r}$ = tamaño del efecto de Rosenthal para Wilcoxon-U Mann-Whitney. 


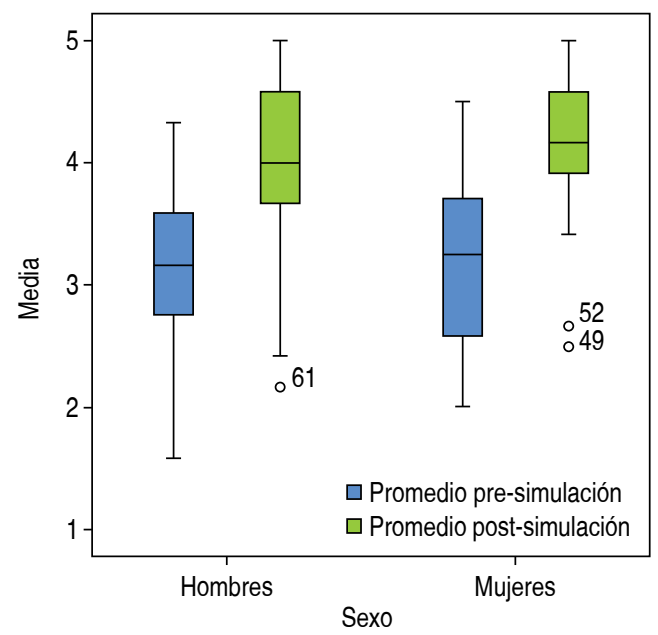

Figura 1: Nivel de confianza global pre-simulación y postsimulación por sexo.

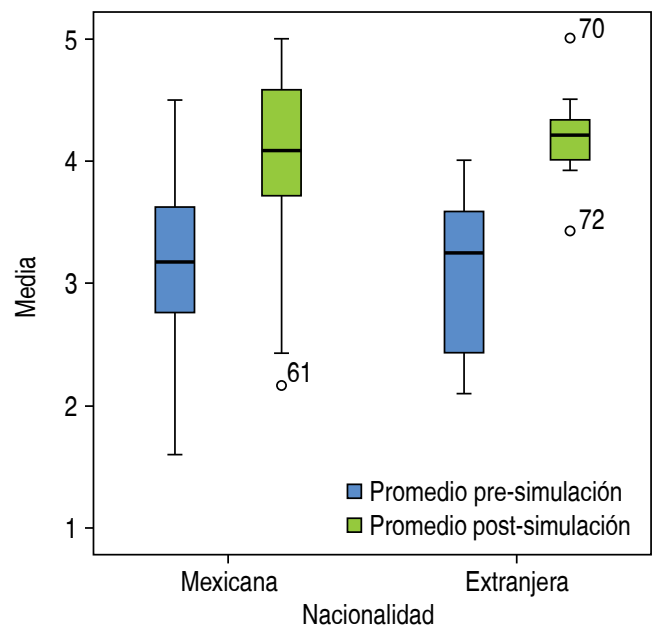

Figura 2: Nivel de confianza global pre-simulación y postsimulación por nacionalidad.

tigación en estudiantes de enfermería, quienes se enfrentaron a la evaluación y manejo de pacientes críticos, concluye que la puntuación en el nivel de confianza después de la simulación aumentó de manera significativa. ${ }^{14}$

Un análisis hecho en médicos internos de pregrado, donde fueron enfrentados a siete escenarios, mostró que conforme realizaron cada uno éstos, fue mejorando el porcentaje de logro promedio. Primero hicieron un caso clínico, luego un debriefing apoyado con video y revisión de los errores, mientras que el siguiente sirvió para evaluar lo aprendido y corregido. Siguiendo la curva de aprendizaje de este grupo al quinto escenario, es decir, después de haber realizado cuatro escenarios con sus respectivos debriefing, llegaron a 90\% de logro sin errores críticos. Posterior a esto, la curva se aplanó con pequeñas mejoras. Esto se justifica, ya que a través de los casos clínicos y debriefing, los estudiantes viven sus errores y experiencias, lo que hace el aprendizaje más significativo. ${ }^{15}$

Es interesante observar que al inicio, la media en el nivel de confianza global de los alumnos extranjeros era menor comparada con los alumnos mexicanos; sin embargo, después de haber tenido las prácticas de simulación, se reflejó un aumento del nivel de confianza, y llegó a ser similar al de los alumnos de origen mexicano; esto puede deberse a diversos factores que dificultan a los extranjeros el aprendizaje teórico, como el idioma, comprensión lectora o contexto sociocultural. Pero, después de la experiencia múltiple en escenarios de simulación, los extranjeros llegan a mejorar y aumentar su nivel de confianza, por lo que se recomienda realizar estudios que puedan explicar este fenómeno.

Por otro lado, la literatura que apoya el aumento en la confianza después de la simulación clínica es muy escasa, la mayoría está hecha por estudiantes de la carrera de enfermería. Por lo tanto, recomendamos incluir la simulación clínica en todas las escuelas de medicina, además de introducirse en diferentes ramas, no solamente en el área de cardiología.

\section{CONCLUSIONES}

Realizar las prácticas de simulación clínica contribuye a la mejora del nivel de confianza en los estudiantes al momento de evaluar a un paciente. Esto puede deberse a la adquisición de los conocimientos y el desenvolvimiento que consiguen al realizar tanto las prácticas como el estudio teórico, ya que una vez que el alumno obtiene conocimiento, aumenta su seguridad para realizar alguna intervención médica.

\section{AGRADECIMIENTOS}

Al Departamento de Apoyo a la Investigación en Ciencias de la Salud, por su ayuda desinteresada en el proceso de implementación de este estudio.

\section{REFERENCIAS}

1. Urra E, Sandoval S, Irribarren F. El desafío y futuro de la simulación como estrategia de enseñanza en enfermería: artículo de revisión. Inv Ed Med. 2017; 6 (22): 119-125 
2. Dávila A. Simulación en Educación Médica: artículo de revisión. Inv Ed Med. 2014; 3 (10): 100-105.

3. Fernández SB. El error médico como causa de muerte. Boletín CONAMED. 2016; 31-26.

4. Fernández SB. Los eventos adversos y la seguridad del paciente. Boletín CONAMED. 2015; 3-9.

5. Lizarazo CF. Simuladores para la enseñanza de la medicina o simulación de la enseñanza. Horizonte Médico. 2012; 12: 6-7.

6. García E, Sibilla JA. Satisfacción de estudiantes de medicina con un Hospital Simulado en Universidad del Valle de México. Horizonte Sanitario. 2016; (15): 135-141.

7. Valencia JL, Tapia S, Olivares SL. La simulación clínica como estrategia para el desarrollo del pensamiento crítico en estudiantes de medicina. Inv Ed Med. 2016.

8. Jones F, Passos-Neto C, Melro BO. Simulation in medical education: brief history and methodology. Principles and Practice of Clinical Research. 2015; 1 (2): 56-63.

9. González MS, García PA. Evaluación de la calidad de dos modelos de simulación clínica. Red de Revistas Científicas de América Latina y el Caribe, España y Portugal. 2016; 11: 677-690.

10. Gordon M. Developments in the use of simulators and multimedia computer systems in medical education. Medical Teacher. 1999; 21 (1): 32-36.

11. Ewy G, Felner J, Juul D, Mayer J, Sajid A, Waught R. Test of a cardiology patient simulator with students in fourthyear electives. J Med Educ. 1987; 62 (9): 738-743.
12. Thomas C, Mackey E. Influence of a clinical simulation elective on baccalaureate nursing student clinical confidence. J Nurs Educ. 2012; 51 (4): 236-239.

13. Mancilla A, De la Hoz PA, Ortiz CP, Vivallos MJ, Del Pilar B. Impacto de la simulación clínica en el nivel de confianza adquirido según características académicas y sociodemográficas de los estudiantes de tercer año de enfermería de la Universidad del Rio Chillan. Universidad del Bío-Bío. Red de Bibliotecas. 2014 (1) 1-130.

14. Mould J, White H, Gallagher R. Evaluation of a critical care simulation series for undergraduate nursing students. Contemporary Nurse. 2011; 38 (1-2): 180-190.

15. Ávila R, Mahana P, Rivera C, Mc Coll P. Simulación Clínica como método de formación de competencias en estudiantes de medicina. Rev Educ Cienc Salud. 2016; 13 (1): 11-14.

Correspondencia:

Verenice Zarahí González-Mejía

Escuela de Medicina de la Universidad de Montemorelos.

Av. Libertad 1300 Pte., Universidad de

Montemorelos, Nuevo León.

Tel. 8262630900, ext. 4005

E-mail: daics@um.edu.mx

1120258@alumno.um.edu.mx 